\title{
Estudio sobre la prevención quimioterapéutica de la caries dental con barnices de clorhexidina y timol, en niños de 5-8 años de edad, con riesgo alto de caries. Un reporte preliminar
}

\author{
García-Santos María C*, Rioboo-García R**
}

\section{RESUMEN}

Objetivos: el propósito de este estudio fue evaluar la posibilidad de reducir la caries de fisura en las caras oclusales de los primeros molares permanentes, a través de barnices antimicrobianos, en escolares con alta incidencia de caries. Se ha realizado un ensayo clínico aleatorio a doble ciego. Sujetos: 35 niños saludables con edades comprendidas entre los 6 y 8 años, con alto riesgo de caries, fueron seleccionados en un colegio de Madrid. Par ser incluidos en el estudio cada niño debería tener como mínimo dos primeros molares permanentes sanos, y presentar caries en dentina en sus molares temporales. Métodos: después de la profilaxis dental, el grupo de estudio se le aplicaba un barniz de clorhexidina $1 \%$ y timol $1 \%$ (Cervitec), y al grupo control un barniz de placebo. El barniz era colocado sobre todos los dientes (dentición temporal y permanente) cada tres meses (al inicio a los 3,6 y 9 meses) y el incremento de caries era comparado a los 12 meses. Resultados: al inicio del estudio no habían diferencias entre los grupos. Al año existía una diferencia significativa entre el grupo de estudio y el de control, en CAO-D P $=0,001$, CAO-S P=0,000 (en los primeros molares permanentes). Conclusión: la aplicación de Cervitec cada 3 meses, es efectiva previniendo la caries en los primeros molares permanentes de escolares con alta actividad de caries.

Palabras clave: Clorhexidina, timol, barniz, alto riesgo de caries, primeros molares permanentes.

\begin{abstract}
Objective: the purpose of this study, was to evaluate the possibility of reducing occlusal fissures caries development, in permanent first molars, using an antimicrobial varnish, in schoolchildren with high caries activity. Randomised, a double-blind clinical trial was developed. Subjects: 35 healthy children aged 6-8 years with high caries risk, were selected from a schools in Madrid. To be included in the study, each child had to have least 2 sound permanent molars and primary molars with carious lesions in dentin. Methods: after prophylaxis, test groups received $1 \%$ chlorhexidine and $1 \%$ thymol varnish (Cervitec) application, the control group received a placebo varnish. The varnish was reapplied in all the teeth (primary and permanent dentitions) every 3 months (initially and after 3,6 and 9 months), and the caries increments were compared at 12 months. Results: no differences between groups were seen at baseline. At year there was a significant difference between test group and control in DMFT P=0.001 DMFS $P=0.000$, (in permanent first molars). Conclusion: the application of Cervitec every 3 months, is effective in preventing caries in permanent first molars, in schoolchildren with high caries activity.
\end{abstract}

Key words: chlorhexidine, thymol, varnish, high caries risk, permanent first molars.

Aceptado para publicación: octubre 2003. 


\section{* Licenciada en odontología. Doctorando del Departamento IV Estomatología.Facultad de Odontología U.C.M. \\ ** Catedrático de Odontología Preventiva y Comunitaria, Departamento IV Estomatología. Facultad de Odontología U.C.M.}

García-Santos María C, Rioboo-García R. Estudio sobre la prevención quimioterapéutica de la caries dental con barnices de clorhexidina y timol, en niños de 5-8 años de edad, con riesgo alto de caries. Un reporte preliminar.. Av. Odontoestomatol 2004; 20-1: 41-53.

\section{INTRODUCCIÓN}

Hoy en día la caries dental se mantiene dentro de las tres enfermedades más comunes en el mundo'; para evitar su aparición es necesario adoptar medidas preventivas, y de esta forma tratar la enfermedad, no los síntomas²; esto se podría lograr suprimiendo los microorganismos que contribuyen especialmente en la formación de caries ${ }^{3}$; como son el Streptococcus mutans (SM) y en ciertas circunstancias, el Lactobacillus (LB), presentes en la placa y en la sali$\mathrm{va}^{4-13}$, no se ha encontrado una correlación igual entre la prevalencia de caries y otras especies microbianas, exceptuando a los Actinomyces en las caries de raíz ${ }^{46}$. Los SM presentan un potencial de producción de caries muy superior al de cualquier microorganismo acidogénico de la placa supragingival ${ }^{4,5,11,14}$. Los SM disminuyen el pH del esmalte de 6 a 5, en 13 minutos, mientras los LB, no lo logran ni siquiera después de unos días ${ }^{15}$. Por otro lado los LB constituyen una fracción muy pequeña de la flora de la placa ${ }^{4,5,15}$, y su tiempo de generación también es más largo ${ }^{4,15}$. El potencial patógeno de SM en la etiología de la caries dental está bien documentado $4,5,7,11,13$ - 17,19 ${ }^{-20}$. Luego la supresión de SM es un factor predominante en la prevención de la caries dental. La primera fuente de infección de SM suele ser la madre ${ }^{21-27}$; si el SM se establece tempranamente en la boca de un niño, éste desarrollará una gran cantidad de caries en sus dientes temporale ${ }^{14,28,29}$; la diferencia en el índice de superficies cariadas y obturadas, entre un niño que presente SM antes de los dos años de edad y la de otro que no lo tenga hasta los cuatro años, es de 10.6 y de 0.3 respectivamente ${ }^{28}$. Estas observaciones ilustran el valor de la prevención temprana del SM. Si se elimina o retrasa esa colonización de SM en la profundidad de las fosas y fisuras, otras bacterias menos odonto-patógenas podrían ocupar ese nicho ecológico, y este antagonismo bacteriano dificultaría la recolonización, y de este modo el desarrollo de la caries ${ }^{14,21,30,31}$ - 34, y dada la baja velocidad de transmisión del $\mathrm{SM}^{35}$, su reducido número en la superficie del epitelio bucal y en saliva, el modo tan localizado de colonización ${ }^{5}$, y su alta susceptibilidad a la clorhexidina (CHX), ${ }^{18,36,37}$ es factible el control de este microorganismo a través de quimioterapéuticos como la $\mathrm{CHX}$; que es el agente antibacteriano más efectivo actualmente conocido ${ }^{13,37-48}$ y con unos efectos secundarios que se podrían considerar aceptables ${ }^{49-66}$. La CHX ha demostrado ser efectiva no importando el vehículo, no obstante, nosotros la utilizaremos en barniz, ya que opinamos que es muy útil en niños, dado que su colocación no depende del paciente o del padre del mismo. Hay barnices de CHX en diferentes porcentajes, escogimos el barniz de Cervitec ${ }^{\circledast}$, que ha demostrado ser efectivo reduciendo $S M^{67.72}$ y mantiene su efecto hasta durante tres meses ${ }^{73.76}$; a pesar de contener la $\mathrm{CHX}$ en un porcentaje muy bajo (1\%), además contiene un $1 \%$ de timol, que posee un efecto sinérgico con la $\mathrm{CHX}^{73}$. El timol proviene de la esencia de tomillo, es un alquifenol, y su derivado biyodado es el aristol ${ }^{77}$.

\section{MATERIAL Y MÉTODO}

\section{POBLACIÓN DE ESTUDIO Y CRITERIOS DE SELECCIÓN}

Para la realización del presente estudio clínico, se revisaron todos los niños de un colegio de Madrid, con edades comprendidas entre 5 y 8 años, en los periodos escolares 2000/2001 (alrededor de 5.00 niños). De los niños revisados, sólo 76 reunieron las siguientes condiciones: -Presentar los cuatro primeros molares permanentes con las caras oclusales completamente erupcionadas. Estas caras oclusales se dividieron en mesial y distal y sólo podían participar los que presentasen menos de cuatro de estos 
ocho sitios, careados, sellados o restaurados. Presentar caries cavitadas clínicamente evidentes en los dientes temporales que podían ser, de primera aparición o de recidiva. 50 niños accedieron a participar; todo acorde con los protocolos de ética estándar.

\section{DISEÑO DEL ESTUDIO}

Estudio experimental, aleatorio, estratificado, a doble ciego; realizado en niños con alta incidencia de caries en sus dientes temporales; se registró el estado bucal inicial y un año después. En la primera visita y cada tres meses se realizó una profilaxis sobre todas las superficies dentales presentes en la boca, seguida del recubrimiento de estas superficies por un barniz de placebo o de tratamiento según el azar. Una persona ajena al operador, preparó unas bolsas con auto cierre, en las que había dos botellitas físicamente idénticas, de color ámbar, con 1.5 $\mathrm{ml}$ de un líquido transparente. Debido a que un pequeño grupo de niños presentaba una cantidad o calidad de caries algo mayor que el resto, se optó por estratificar el grupo y así evitar un error por asignación, que podría comprometer la validez interna del estudio. Tomando en cuenta lo anterior nos prepararon un grupo de bolsas para los de alto riesgo de caries, en las que el $50 \%$ de las bolsas era placebo y el resto de ellas tratamiento y de igual modo para los de riesgo normal (todos los niños eran de alto riesgo, esta denominación era comparativa). De esta forma, había dos recipientes, uno de alto riesgo y otro de riesgo normal donde los niños tomaban de forma aleatoria la bolsa, dependiendo a qué grupo perteneciesen ellos. Las bolsas presentaban un código, junto con las bolsas nos entregaron dos sobres lacrados (para abrirlos una vez finalizado el estudio), con la información de qué códigos de las bolsas era el tratamiento y cuales al placebo. El barniz de tratamiento, Cervitec ${ }^{\circledast}$ (Ivoclar-Vivadent, Schaan, Liechtenstein), contiene dos antimicrobianos: clorhexidina $1 \%$ y timol $1 \%$, un polímero: polivinilbutirol $10 \%$ y un solvente: etanol-etilacetato $88 \%$. El barniz de placebo contiene: un polímero, que es polivinilbutirol y un solvente, el etanol-etilacetato; con las mismas características organolépticas para los cinco sentidos, que el barniz del Cervitec ${ }^{\circledast}$.

\section{EXAMEN CLÍNICO Y COLOCACIÓN DE LOS BARNICES}

El estudio de llevó a cabo en una habitación completamente acristalada con luz natural y fluorescentes en el techo, que tenía una camilla articulada, una silla con ruedas para el operador y una mesa-bandeja también con ruedas, una unidad odontológica portátil completa (ultrasonido, pieza de mano con contra-ángulo y jeringa de tres funciones), una lámpara para la unidad, un equipo de alta succión, así como todo el instrumental y consumibles necesarios para el desarrollo del estudio. Con el objeto de lograr un criterio único de estimación de caries, el examen buco-dental así como la aplicación de los barnices, fue realizado exclusivamente por el autor. El examen clínico se comenzaba con la profilaxis dental, con ultrasonido si el paciente presentaba sarro, y pulido de las superficies con piedra pómez y cepillo montado en pieza de mano con contra-ángulo. A medida que se iban limpiando los dientes se procedía a su aislamiento con un retractor de labios, un abre-boca, eyector y rollos de algodón; con las superficies dentales limpias, secas y el campo bien aislado, se realizaba la inspección visual con un espejo dental planos y un explorador de punta fina, sin presión, y el rellenado del odontograma; las anotaciones de los datos dentales se registraban según los criterios de caries de la $\mathrm{OMS}^{78}$. Sin retirar el aislamiento se colocaba el abre-bocas del lado izquierdo y se aplicaba el barniz en el lado derecho, superior e inferior, que estaban perfectamente aislados con el retractor de labios y los rollos de algodón; el barniz era aplicado en todas las superficies dentales, con un colocador suministrado por el fabricante; primero se hacía una aplicación muy delgada del barniz, para que se fuese secando a medida que lo íbamos colocando en otros dientes, y este procedimiento se repetía tres veces, se esperaba a que el barniz se fraguase e inmediatamente cambiábamos el abre-boca de lugar y repetíamos este mismo procedimiento en el lado contrario. Una vez finalizado esto, se les daban a los niños unas instrucciones verbales y por escrito de no enjuagarse ni comer o ingerir algún líquido hasta que llegasen a casa (tres horas después) y de no cepillarse los dientes ese día. La profilaxis dental y la colocación de los barnices se repitió a los tres, seis y nueve meses, el registro del odontograma sólo se realizó la primera cita y un año des- 
pués, y en esa oportunidad cualquier indicio de caries, incluso las incipientes, eran registradas. Una vez finalizado el estudio se sellaron todos los primeros molares permanentes que se encontraban ilesos y los que presentaban caries se eliminaron éstas y restauraron.

\section{ANÁLISIS ESTADÍSTICO}

El análisis estadístico de los datos se realizó en el Departamento de Apoyo a la Investigación, de los Servicios Informáticos de la U.C.M., utilizando el programa SPSS 11.5 para Windows. Los métodos estadísticos utilizados fueron los siguientes (SPSS, 2002) ${ }^{79}$ : Test de la $t$ de Student pareada (procedimiento T-TEST) para la comparación muestras relacionadas comparando las medias de un atributo en dos momentos del tiempo (antes y después) ${ }^{80}$. Análisis de la varianza, ANOVA (procedimiento GLM) para el análisis de varianza para variables dependientes múltiples con un factor (grupo ${ }^{80}$. Análisis de medidas repetidas (procedimiento GLM) para el análisis del comportamiento de grupos de un factor inter-sujetos (grupo) en las medidas de dientes y superficie en un factor intra-sujeto (tiempo, antes y después) ${ }^{80}$.

\section{RESULTADOS}

El estudio finalizó con 35 niños, de los cuales 18 eran placebo y 17 Cervitec $^{*}$.
Se designó el nivel de significancia del 95\% como estadísticamente significativo.

1. Al comenzar el estudio no existían diferencias estadísticamente significativas entre los grupos placebo y Cervitec $^{\oplus}$ (tabla 1).

2. En un año se desarrollaron nuevas lesiones de caries, siendo esto más evidente en los molares permanentes, que en los temporales (tabla 2).

3. Existe una diferencia estadísticamente significante en el menor desarrollo de la enfermedad entre los dientes permanentes tratados con Cervitec ${ }^{\circledast}$, con respecto a los tratados con placebo tanto en las medidas de diente (CAO-D) como de superficie (CAO-S), en dientes permanentes; esto se puede apreciar en la tabla 3, así como en los gráficos 3 y 4.

\section{DISCUSIÓN}

Nuestros resultados sugieren:

Primero: Que en un año es posible que se desarrollen nuevas lesiones de caries en las caras oclusales de los primeros molares permanentes en niños de seis a ocho años de edad con alto riesgo de caries (tabla 2).

Este desarrollo tan notorio de las caries puede ser consecuencia de que la muestra escogida presentaba simultáneamente varias características primordiales para la formación de caries, tales como:

\begin{tabular}{|c|c|c|c|c|c|}
\hline \multicolumn{6}{|c|}{$\begin{array}{l}\text { TABLA 1.- ANOVA DE UN FACTOR INDEPENDIENTE GRUPO PLACEBO Y CERVITEC }{ }^{\circledR} \text {; } \\
\text { EN DIENTES TEMPORALES Y PERMANENTES, EN EL MOMENTO INICIAL DEL ESTUDIO. }\end{array}$} \\
\hline $\begin{array}{l}\text { DIENTES } \\
\text { INICIO DEL ESTUIDIO }\end{array}$ & GRUPO & $N$ & $M E D I A \pm D S$ & $F$ & $P$ \\
\hline \multirow[t]{2}{*}{ cao-d } & PLACEBO & 18 & $5,890 \pm 2,610$ & \multirow[t]{2}{*}{0,706} & \multirow[t]{2}{*}{$0,407 \mathrm{NS}$} \\
\hline & CERVITEC & 17 & $6,590 \pm 2,293$ & & \\
\hline \multirow[t]{2}{*}{ cao-s } & PLACEBO & 18 & $9,444 \pm 5,216$ & \multirow[t]{2}{*}{1,001} & \multirow[t]{2}{*}{$0,324 \mathrm{NS}$} \\
\hline & CERVITEC & 17 & $11,765 \pm 8,250$ & & \\
\hline \multirow[t]{2}{*}{ CAO-D } & PLACEBO & 18 & $0,944 \pm 1,349$ & \multirow[t]{2}{*}{1,266} & \multirow[t]{2}{*}{$0,269 \mathrm{NS}$} \\
\hline & CERVITEC & 17 & $0,471 \pm 1,125$ & & \\
\hline \multirow[t]{2}{*}{ CAO-S } & PLACEBO & 18 & $1,167 \pm 1,618$ & \multirow[t]{2}{*}{2,159} & \multirow[t]{2}{*}{$0,151 \mathrm{NS}$} \\
\hline & CERVITEC & 17 & $0,471 \pm 1,125$ & & \\
\hline
\end{tabular}


TABLA 2.- ANOVA DE UN FACTOR PAREADO, FACTOR TIEMPO: ANTES, DESPUÉS; EN DIENTES TEMPORALES Y PERMANENTES.

\begin{tabular}{|c|c|c|c|c|c|c|}
\hline DIENTES & $N$ & $\begin{array}{c}\text { ANTES } \\
M E D I A \pm D S\end{array}$ & $N$ & $\begin{array}{c}\text { DESPUÉS } \\
M E D I A \pm D S\end{array}$ & $T$ & $P$ \\
\hline cao-d & 35 & $6,230 \pm 2,451$ & 35 & $6,400 \pm 2,580$ & $-1,642$ & $0,110 \mathrm{NS}$ \\
\hline cao-s & 35 & $10,571 \pm 6,857$ & 35 & $11.310 \pm 7,319$ & $-2,242$ & $0,032 \mathrm{~S}$ \\
\hline CAO-D & 35 & $0,714 \pm 1,250$ & 35 & $1,890 \pm 1,843$ & $-4,384$ & $0,000 \mathrm{~S}$ \\
\hline CAO-S & 35 & $0,829 \pm 1,424$ & 35 & $3,200 \pm 3,333$ & $-4,853$ & $0,000 \mathrm{~S}$ \\
\hline
\end{tabular}

TABLA 3.- ANOVA DE DOS FACTORES: FACTOR TIEMPO PAREADO ANTES-DESPUÉS Y FACTOR TRATAMIENTO INDEPENDIENTE ENTRE LOS GRUPOS PLACEBO Y CERVITEC ${ }^{\circledR}$, EN DIENTES TEMPORALES Y PERMANENTE.

\begin{tabular}{|c|c|c|c|c|c|c|}
\hline DIENTE & GRUPO & TIEMPO & $N$ & $M E D I A \pm D S$ & $F$ & $P$ \\
\hline \multirow[t]{4}{*}{ Cao-d } & \multirow[t]{2}{*}{ PLACEBO } & ANTES & 18 & $5,890 \pm 2,610$ & \multirow[t]{4}{*}{1,102} & \multirow[t]{4}{*}{$0,301 \mathrm{NS}$} \\
\hline & & DESPUÉS & 18 & $6,170 \pm 2,792$ & & \\
\hline & \multirow[t]{2}{*}{ CERVITEC } & ANTES & 17 & $6,590 \pm 2,293$ & & \\
\hline & & DESPUÉS & 17 & $6,650 \pm 2,396$ & & \\
\hline \multirow[t]{4}{*}{ Cao-s } & \multirow[t]{2}{*}{ PLACEBO } & ANTES & 18 & $9,444 \pm 5,216$ & \multirow[t]{4}{*}{2,300} & \multirow[t]{4}{*}{$0,139 \mathrm{NS}$} \\
\hline & & DESPUÉS & 18 & $10,670 \pm 6,334$ & & \\
\hline & \multirow[t]{2}{*}{ CERVITEC } & ANTES & 17 & $11,765 \pm 8,250$ & & \\
\hline & & DESPUÉS & 17 & $12,000 \pm 8,382$ & & \\
\hline \multirow[t]{4}{*}{ CAO-D } & \multirow[t]{2}{*}{ PLACEBO } & ANTES & 18 & $0,944 \pm 1,349$ & \multirow[t]{4}{*}{14,104} & \multirow[t]{4}{*}{$0,001 \mathrm{~S}$} \\
\hline & & DESPUÉS & 18 & $2,940 \pm 1,697$ & & \\
\hline & \multirow[t]{2}{*}{ CERVITEC } & ANTES & 17 & $0,471 \pm 1,125$ & & \\
\hline & & DESPUIÉS & 17 & $0,760 \pm 1,251$ & & \\
\hline \multirow[t]{4}{*}{ CAO-S } & \multirow[t]{2}{*}{ PLACEBO } & ANTES & 18 & $1,167 \pm 1,618$ & \multirow[t]{4}{*}{26,643} & \multirow[t]{4}{*}{$0,000 \mathrm{~S}$} \\
\hline & & DESPUIÉS & 18 & $5,389 \pm 3,183$ & & \\
\hline & \multirow[t]{2}{*}{ CERVITEC } & ANTES & 17 & $0,471 \pm 1,125$ & & \\
\hline & & DESPUIÉS & 17 & $0,882 \pm 1,317$ & & \\
\hline
\end{tabular}

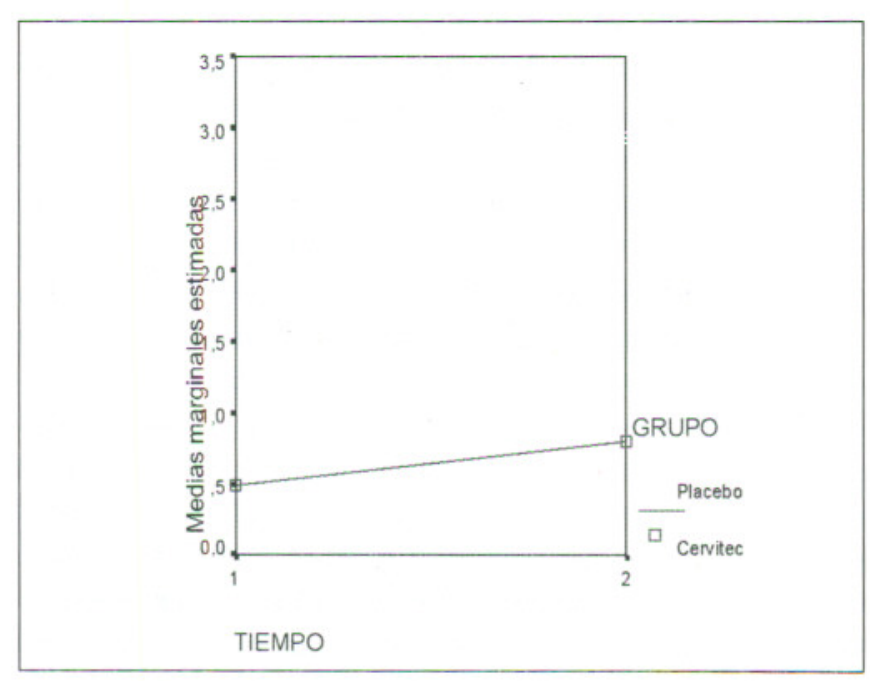

Gráfico 1 CAO-D

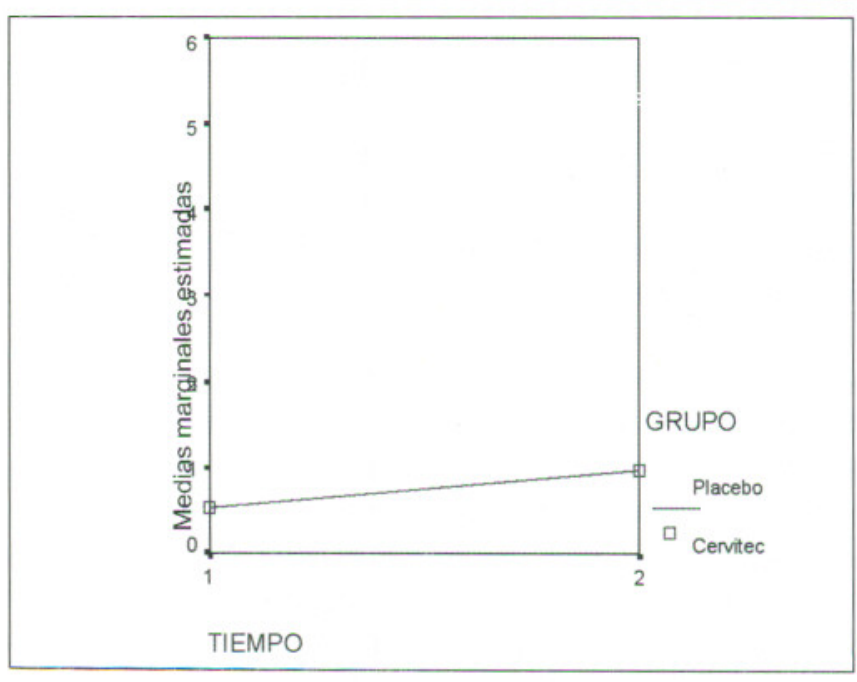

Gráfico 2 CAO-S 
1. Los niños seleccionados tenían altos valores en los índices de caries en sus dientes temporales, así como un gran número de lesiones sin tratar, y esto es una fuente de infección de bacterias cariógenas ${ }^{81}$. Los dientes primarios son la fuente de infección más probable para los dientes permanentes ${ }^{9}$. La caries en los dientes temporales puede ser usada como un indicador de riesgo, para predecir caries en los dientes permanentes ${ }^{13,82-84}$, y nuestros niños tenían el triple de posibilidades de desarrollar caries en sus dientes permanentes con respecto a niños sin caries en sus temporales ${ }^{84}$. Las fosas y fisuras de sus primeros molares recién erupcionado eran un lugar idóneo para que el SM se alojase en ellas.

2. El grupo lo formaban niños pequeños, la edad del niño predice la habilidad en el cepillado ${ }^{85}$, y la placa bacteriana se forma y mantiene en las fisuras de las caras oclusales sin ser afectada por la autoclisis ${ }^{15}, y$ en ausencia de higiene bucal y con una exposición frecuente a alimentos ricos en sacarosa, es fácil dar lugar a lesiones incipientes en tan sólo 3 semanas ${ }^{42,86}$; más aún si hablamos de pacientes de alto riesgo.

3. La superficie dental escogida para el estudio era la cara oclusal, que es la superficie en la que se desarrolla más caries en un diente $e^{5,87-91}$. También estábamos trabajando con los dientes que presentan más tendencia a la caries ${ }^{91,92}$.

4. Los dientes recién erupcionados (esmalte inmaduros) son más susceptibles a las caries $^{90,93-97}$.

Éste cúmulo de circunstancias pudo ser el causante de un mayor número de caries, estadísticamente significativo, en los primeros molares que no fueron tratados con Cervitec $^{\circledast}$, dado que el alto riesgo hace que se exprese de forma más evidente el efecto protector del producto ${ }^{3,98-101}$.

Segundo: Que un barniz que contiene clorhexidina y timol, ambos al $1 \%$ (Cervitec $\left.{ }^{\circledast}\right)$, colocado trimestralmente, en cuatro ocasiones (al inicio, a los tres, seis y nueve meses), puede ser efectivo aminorando la formación de caries en las caras oclusales de los primeros molares permanentes durante los periodos inmediatos a su erupción, en niños con gran tendencia a la caries (tabla 3 y gráficos 1 y 2 ).
Loesche ya en 1983(3), observó que el tratamiento eficaz de algunos antimicrobianos como los antibióticos, el flúor y la clorhexidina, podía deberse más, a que se habían escogido pacientes con alto riesgo de caries, y no a la acción antibacteriana de una u otra droga ${ }^{3}$. Esto vendría a explicar por qué en muchos estudios no se logró demostrar la efectividad de la clorhexidina en la prevención de la caries, a pesar de que en el control bacteriológico, cuando se llevó a cabo en algunos de esos estudios, sí se evidenció la disminución de las concentraciones bacterianas en placa o saliva $a^{7,13,91,102 \cdot 104}$. Un ejemplo elocuente de esa observación de Loesche serían los trabajos de: Zickert, Emilson y Krasse en $1982^{(99)}$, después de tres años utilizando un gel de clorhexidina al $1 \%$, observaron una reducción del $80 \%$ en la incidencia de caries en los pacientes de alto riesgo, sin obtener los mismos resultados, en los pacientes que no eran de alto riesgo ${ }^{99}$. Los mismos autores, en 1987(100), encontraron que los tratamientos con antimicrobianos lograban una marcada reducción de la caries, especialmente en niños con más de $10^{6}$ SM por ml de saliva ${ }^{100}$. Fennis-le YL y cols $(1998)^{101}$ colocaron barnices de clorhexidina al $40 \%$ o placebo y no observaron diferencias significativa entre los grupos de placebo y clorhexidina, situación que cambió cuando se estratificaron los grupos en alto y bajo riesgo ${ }^{101}$. Otra de las razones que posiblemente determinaron esta elevada reducción de caries en las caras oclusales de los primeros molares permanentes tratados con barnices de Cervitec ${ }^{\circledast}$, se debiese a la interferencia bacteriana en la recolonización de SM. Cuando realizamos la profilaxis dental de forma prolija, acto que se repitió antes de cada colocación trimestral de los barnices, posiblemente hayamos logrado retirar gran parte de los SM de sus nichos ecológicos; una vez retiradas las bacterias de sus nichos, éstos volverían a ser recolonizados de forma muy similar a la preexistente y en un corto plazo de tiempo, debido a que se mantenían las mismas condiciones iniciales ideales para el desarrollo de esa flora bacteriana, y posiblemente habrá ocurrido así en los pacientes tratados con el barniz placebo. Sin embargo en el grupo tratado con el barniz de Cervitec ${ }^{\circledast}$, esto no se daría de igual modo, debido a que las bacterias involucradas en los procesos de caries, son muy sensibles a la clorhexidina, especialmente los $\mathrm{SM}^{18,36,37}$, que con un corto contacto del barniz de clorhexidina sobre los dientes puede 
ser suficiente para reducirlos ${ }^{105}$. Todo lo anterior nos lleva a pensar que no sólo tendremos el efecto bactericida y posteriormente bacteriostático de la clorhexidina de forma más duradera de lo considerado normal (por su condición de barniz), sino que además podríamos contar con el antagonismo bacteriano en la competición por el nicho ecológico ${ }^{30-34}$, en este caso con ventaja para el $S$. sanguis que incrementa su número después de la aplicación de clorhexidina $^{53,60}$; por otro lado, el SM no se disemina rápidamente de unos dientes a otros ${ }^{35,89}$ dadas sus reducidas concentraciones salivales disponibles para la adherencia y su dificultad para absorberse a los dientes ${ }^{5,6}$, en cambio el S. sanguis tiene mejor capacidad para adherirse a las superficies denta${ }^{l e s}{ }^{6,106}$ y es más numerosos en la placa ${ }^{6}$, además de tener capacidad para producir bacteriocinas como la sanguicina, que es inhibidora de $\mathrm{SM}^{107}$. El A. naeslundii también inhibe el recrecimiento del SM después de la supresión de este último con la clorhexidina ${ }^{31}$, y la clorhexidina no tiene ningún efecto significativo en el número de Actinomyces naeslundii 56,57. Todo lo anterior le obstaculizará de forma considerable al SM la recolonización dental, dificultando así el desarrollo de la caries. Después de este tratamiento intensivo con clorhexidina, se restablece una microflora oral normal, caracterizada por una baja proporción de $\mathrm{SM}^{30}$, y de esta forma la mala higiene oral, así como la ingesta descontrolada de sacarosa, no tendrán tantos efectos perniciosos sobre los dientes, porque la ausencia de higiene y la ingesta de sacarosa no produce caries si los dientes estaban regularmente tratados con agentes antibacterianos, como la corhexidina ${ }^{42}$. El efecto del barnices de Cervitec $^{\circledast}$ se ha mantenido hasta por seis meses cuando se ha colocado en dentina ${ }^{108}$; esto se puede deber a que el Cervitec ${ }^{\oplus}$ penetra dentro de la dentina careada hasta 35 micrones ${ }^{109}$, nuestros barnices se colocaron sobre todas las superficies dentales, incluidas las cavidades abiertas de caries (dentina cariada), de los molares temporales; esto aunado al hecho de que la clorhexidina suprime con mayor intensidad el SM a nivel de los premolares ${ }^{110}$ (localización coincidente con los molares temporales), puede haber creado un nivel de clorhexidina superior, y por ende más efectivo, repercutiendo esto positivamente en los primeros molares permanentes de forma indirecta; de hecho, el nivel de reducción de caries conseguido en los primeros molares per- manentes en este estudio, fue superior a los logrados en otros trabajos similares. En la literatura científica existe una gran cantidad de trabajos en los que se han utilizado los barnices de Cervitec $^{\circledast}$ para prevenir la caries dental, no obstante, no hay muchos estudios que hayan podido demostrar significativamente esta prevención de caries.

\section{CONCLUSIONES}

En base a los resultados obtenidos inferimos las siguientes conclusiones:

1. Los barnices que contienen clorhexidina al $1 \%$ y timol al $1 \%$, Cervitec ${ }^{\oplus}$ aplicados en niños con gran tendencia a la caries, trimestralmente en cuatro oportunidades (en el inicio, a los tres, seis y nueve meses), son efectivos aminorando la formación de caries en las caras oclusales de los primeros molares permanentes, durante los periodos inmediatos a su erupción.

2. En un año es posible que se desarrollen nuevas lesiones de caries en las caras oclusales de los primeros molares permanentes en niños de seis a ocho años de edad con alto riesgo de caries, si no se toman medidas preventivas.

3. No hemos observado en ninguno de los niños pigmentaciones ni dentales ni de la lengua, durante el año que duró el estudio, debidas a la colocación trimestral de los barnices de Cervitec ${ }^{\oplus}$.

4. No se apreciaron erosiones en las mucosas, no obstante, sería interesante que se mejorase esa sensación de ardor en las mucosas y olor muy penetrante, que hemos encontrado en la clínica de este barniz, para poderlo colocar en niños incluso de menor edad.

5. Se debería intentar prolongar la acción del producto hasta seis meses, para volver más práctico y económico su uso de forma masiva.

6. De todo lo anterior podemos concluir que los barnices de Cervitec ${ }^{\circledR}$, en nuestra opinión, se podrían considerar como otra opción más en la prevención de las caries, de las caras oclusales en los niños con alto riesgo de sufrir esta enfermedad, dado que ellos precisan algo más que los procedimientos estándar utilizados para prevenir la caries. 


\section{BIBLIOGRAFÍA}

1. Anusavice KJ. Dental caries: risk assessment and treatment solutions for an elderly population. Compend Contin Educ Dent. 2002 Oct;23(10 Suppl): 12-20.

2. Anderson MH, Bales DJ, Omnell KA. Modern management of dental caries: the cutting edge is not the dental bur. J Am Dent Assoc. 1993 Jun;124 (6): 36-44.

3. Loesche Waer J. Antimicrobials, Can They Be Effective. In: Cariology Today. Int. Congr., Zürich 1983, pp. 293-300 (Karger, Basel, 1984)

4. MacDonald JB. Microbiology of Caries. In: Sognnaes RF., ed. : Chemistry and the prevention of Dental Caries. C. C. Thomas, Springfield, III., 1962; 89-125.

5. Gibbons RJ and VanHoute J. Bacteriology of Dental Caries. In Shaw JH, Sweeney EA, Cappacino CC and Miller SM. Texbook of Oral Biology. Saunder, Philadelphia, 1978; 975-91.

6. Loesche WJ. Dental caries. A treatable infectión. Chicago: Thomas, 1982.

7. Bowden GH. Mutans streptococci caries and chlorhexidine. Biol Oral 1982; 27 (10): 86168.

8. Zickert I, Emilson CG, Krasse B. Streptococcus mutans, lactobacilli and dental heah in 13-14year-old Swedish children. Community Dent Oral Epidemiol. 1982 Apr;10 (2): 77-81.

9. Loesche WJ. Eklund S. Earnest R. Burt B. Longitudinal investigation of bacteriology of humane fissure decay: epidemiological studies in molars shortly after eruption. Infection $\varepsilon$ Immunity. 1984; 46 (3): 765-72 (abstract).

10. Boyar RM, Bowden GH. The Microflora Associated with the Progression of Incipient Carious Lesions in Teeth of Children Living in a Water-Fluoridated Area. Caries Res 1985; 19 : 298-06.
11. Emilson CG, Krasse B. Support for and implications of the specific plaque hypothesis. Scand $\mathrm{J}$ Dent Res 1985 Apr; 93 (2): 96-04.

12. Krasse B. Biological factors as indicators of future caries. Int Dent J 1988 Dec; 38 (4): 219-25.

13. Bowden GH. Mutans streptococci caries and chlorhexidine. J Can Dent Assoc 199662 (9): 700-07.

14. Loesche WJ. Role of Streptococcus mutans in Human Dental Decay, Microbiol Rev. 1986; 50 (4): 353-80.

15. Strålfors Allan. Investigations into the bacterial chemistry of dental plaque. Odontologisk Tidshrift 58: 155-341, 1950.

16. Loesche WJ. Straffon LH. Longitudinal investigation of the role of Streptococcus mutans in human fissure decay. Infection \& Immunity. 1979; 26 (2): 498-507.

17. Thibodeau EA, O'Sullivan DM, Tinanoff $N$. Mutans streptococci and caries prevalence in preschool children. Community Dent Oral Epidemiol 1993 Oct; 21 (5): 288-91.

18. Riobóo R. Quimioterapia de la Placa Bacteriana. Rev. Española de Estomatol. 1982; (30): 335-52.

19. Nyvad B, Kilian M. Comparison of the initial streptococcal microflora on dental enamel in caries-active and in caries-inactive individuals. Caries Res 1990; 24 (4): 267-72.

20. Twetman S, Petersson LG. Interdental caries incidence and progression in relation to mutans streptococci suppression after chlorhexidine-thymol varnish treatments in schoolchildren. Acta Odontol Scand. 1999 Jun; 57 (3): 144-8.

21. Loesche W.J. Nutrition and dental decay in infants. American Journal of Clinical Nutrition. 1985; 41 (2 suppl): 423-35. (abstract).

22. Tenovuo J, Hakkinen P, Paunio P, Emilson CG. Effects of chlorhexidine-fluoride gel treatments in 
mothers on the establishment of mutans streptococci in primary teeth and the development of dental caries in children. Caries Res 1992; 26 (4): 275-80.

23. Caufield PW, Cutter GR and Dasanayake AP. Evidence for a Discrete Window of Infectivity. J Dent Res. 1993; 72 (1): 37-5.

24. Y. Li and P. W. Caufield The fidelity of initial acquisition of mutans streptococci by infants from their mothers. J. Dent. Res. 1995 74: 681-5 (Abstract).

25.Kozai K, Nakayama R, Tedjosasongko U, Kuwahara S, Suzuki J, Okada M, Nagasaka N. Intrafamilial distribution of mutans streptococci in Japanese families and possibility of father-to-child transmission. Microbiol Immunol 1999; 43 (2): 99-06.

26. Smith RE, Badner VM, Morse DE, Freeman K. Maternal risk indicators for childhood caries in an inner city population. Dent Oral Epidemiol 2002 jun; 30 (3): 176-81.

27. Tedjosasongko $U$, Kozai K. Initial acquisition and transmission of mutans streptococci in children at day nursery. ASDC J Dent Child 2002 SepDec; 69 (3): 284-8, 234-5.

28. Alaluusua S, Renkonen OV. Streptococcus mutans establishment and dental caries experience in children from 2 to 4 years old. Scand J Dent Res 1983 Dec; 91 (6): 453-7.

29. Khöler B, Andreen I., Johnson B. The earlier the colonization by streptococci, the higher the caries prevalence at 4 years of aged. Oral Microbiol Immunol. 1988; 3: 14-7.

30. Schaeken MJ, van der Hoeven JS, van den Kieboom CW. Effect of chlorhexidine varnish on streptococci in dental plaque from occlusal fissures. Caries Res.1994; 28 (4): 262-6.

31. Van der Hoeven JS, Schaeken MJ. Streptococci and actinomyces inhibit regrowth of Streptococus mutans on gnotobiotic rat molar teeth after chlorhexidine varnish treatment. Caries Res 1995; 29 (2): 159-62.

32. Caufield PW, Dasanayake AP, Li Y, Pan Y, Hsu J, Hardin JM. Natural history of Streptococcus sanguinis in the oral cavity of infants: evidence for a discrete window of infectivity. Infect Immun 2000 Jul; 68 (7): 4018-23.

33. Baca García P. Fundamento, desarrollo y unidad de los barnices de clorhexidina en el control de la caries dental: revisión bibliografía I. Chlorzoin y EC40. Archivos de Odonto-Estomatología Preventiva y Comunitaria. 2000; 16 (9): 586-95.

34. Baca García P. Fundamento, desarrollo y unidad de los barnices de clorhexidina en el control de la caries dental: revisión bibliografía II. Cervitec ${ }^{\circledast}$. Archivos de Odonto-Estomatología Preventiva y Comunitaria. 2001; 17 (2): 87-5.

35. Loesche WJ, Svanberg ML,Pape HR. Intraoral Transmission of Streptococcus Mutans by a Dental Explorer. J. Dent. Res. 1979; 58 (8): 1765-70.

36. Balanyk TE, Sandham HJ.Development of sustained-release antimicrobial dental varnishes effective against Streptococcus mutans in vitro. J Dent Res 1985 Dec; 64 (12): 1356-60.

37. Emilson CG. Potential efficacy of chlorexidine against mutans streptococci and human dental caries. J Dent Res 1994; 73 (3): 682-91.

38. Löe Harald and Schiøtt Rindom, The Effect of Mouthrinses and Topical Application of Chlorexidine on the Development of Dental Plaque and Gingivitis in Man, J. Periodont. Res. 1970; 5: 79-3.

39. Schiøtt Rindom C., Löe Harald, Jensen Borglum S., Kilian, M., Davies R. M. and Glavind K., The Effect of Chlorhexidine Mouthrinses on the Human Oral Flora, J. Periodont. Res. 1970; 5: 84-9.

40. Rölla Gunnar, Löe Harald and Schiøtt Rindom C., The Affinity of Chlorhexidine for 
Hydroxyapatite and Salivary Mucins, J. Periodont. Res. 1970; 5: 90-5.

41. Davies, R. M., Borglum Jensen, S., Rindom Schiøtt C., and Loe, H., The Effect of Topical Application of Chlorhexidine on the Bacterial Colonization of the Teeth and Gingiva, J. Periodont. Res 1970; 5:96-01

42. Lóe H, von der Fehr FR, Schiött CR. Inhibition of Experimental Caries by Plaque Prevention. The Effect of Chlorhexidine Mouthrinses. Scand J Dent Res 1972;80: 1-9

43. Addy M. Chlorhexidine compared with other locally delivered antimicrobials. A short review. J Clin Periodontol 1986 Nov;13(10):957-64

44. Adams D, Addy M. Mouthrinses. Adv Dent Res 1994 Jul;8(2):291-301

45. Bascone, A.; Manso, F. J.: Clorhexidina en Odontoestomatología: Conceptos actuales y revisión de la literatura. Avances en Odontoestomatología 1994; 10: 685-08.

46. Quirynen M, Avontroodt P, Peeters W, Pauwels M, Coucke W, van Steenberghe D. Effect of different chlorhexidine formulations in mouthrinses on de novo plaque formation. J Clin Periodontol. 2001 Dec;28(12):1127-36

47. Jones CG. Chlorhexidine: is it still the gold standard? Periodontol 2000.1997;15:55-62.

48. Matthijs S, Adriaens PA. Chlorhexidine varnishes: a review. J Clin Periodontol 2002;29(1):1-8

49. Flötra L, Gjermo P, Rölla G and Waerhaug J. Side effects of chlorhexidine mouth washes. Scand . J. dent. Res. 1971; 79: 119-25

50. Gjermo P, and Eriksen HM. Unchanged plaque inhibiting effect of chlorhexidine in human subjects after two years of continuous use. Archs. Oral Biol. 1974;19:317-319.

51. Löe H, Schiøtt CR, Glavinf L., Karring T. Two Years Oral Use of Chlorhexidine in Man I. General design and clinical effects. J. Periodont. Res 1976;11:135-44.

52. Rindom-Schiøtt C, Löe H \& Brinner WW. Two Year Oral Use of Chlorhexidine in Man IV. Effect on various medical parameters. J. Periodont. Res 1976; 11:158-64.

53. Emilson CG, Fornell J. Effect of toothbrushing with chlorhexidine gel on salivary microflora, oral hygiene, and caries. Scand J Dent Res. 1976 Sep;84(5):308-19.

54. Case DE. Safety of Hibitane. I. Laboratory experiments. J Clin Periodontol 1977 Dec;4(5):66-2

55. Fitzgerald RJ, Fitzgerald DB, Adams BO. Effects of chlorhexidine gluconate in drinking water on dental caries and oral microorganisms in the Syrian hamster. Arch Oral Biol 1986;31(10):7079.

56. Schaeken MJM, Van Der Hoeven JS, Hendriks JCM. Effects of varnishes containing chlorhexidine on the human dental plaque flora. J.Dent Res 1989; 68: 1786-9.

57. Fure S, Emilson CG.Effect of chlorhexidine gel treatment supplemented with chlorhexidine varnish and resin on mutans streptococci and Actinomyces on root surfaces. Caries Res. 1990;24(4):242-7.

58. Schaeken MJ, Kejens HM, Van Der Hoeven JS. Effects of fluoride and chlorhexidine on the microflora of dental root surfaces and progression of root-surface caries. J Dent Res 1991 Feb;70(2):150-3.

59. Steinberg D, Amit U, Brayer L, Sela MN, Friedman $M$. The effect of sustained-release varnish of chlorhexidine in dental plastic shells on salivary Streptococcus mutans. Clin Prev Dent 1991 Mar-Apr;13(2):9-12.

60. Sandham HJ, Brown J, Chan KH, Phillips HI, Burgess RC, Stokl AJ. Clinical trial in adus of an antimicrobial varnish for reducing mutans streptococci. : J Dent Res 1991 Nov;70(11):1401-8 
61. Al-Tannir M. A., Goodman H. S., <A Review of Chlorhexidine and its Use in Special Populations. Spec Care Dent. 1994;14(3):116-22

62. Fan M, HeH, Ling J. The effect of chlorhexidine varnish system on Streptococcus mutans in fissure plaques. Zhonghua Kou Qiang Yi Xue Za Zhi 199732 (5) 269-71

63. van Strijp AJ, van Steenbergen TJ, ten Cate JM. Effects of chlorhexidine on the bacterial colonization and degradation of dentin and completely demineralized dentin in situ. Eur J Oral Sci 1997 Feb;105(1):27-5.

64. Achong, RA, Briskie, DM, Hildebrandt, GH, Feigal, RJ, Loesche WJ. Effect of chlorhexidine varnish mouthguards on the levels of selected oral microorganisms in pediatric patients. Pediatric Dentistry, 1999; 21(3):169-75.

65. Ekenbäck SB, Linder LE, Lonnies H. Effect of four dental varnishes on the colonization of cariogenic bacteria on exposed sound root surfaces. Caries Res. 2000 Jan-Feb;34(1):70-4.

66. Beyth N, Redlich M, Harari D, Friedman M, Steinberg D. Effect of sustained-release chlorhexidine varnish on Streptococcus mutans and Actinomyces viscosus in orthodontic patients. Am J Orthod Dentofacial Orthop 2003 Mar;123(3):345-8

67. Huizinga ED, Ruben J, Arends J. Effect of an antimicrobial-containing varnish on root demineralisation in situ. Caries Res 1990;24(2):130-2.

68. Bratthall D, et al. A study into the prevention of fissure caries using an antimicrobial varnish. Int Dent J 1995;45 (4):245-54.

69. Svante Twetman ; Lars G. Petersson. : Interdental caries incidence and progression in relation to mutans streptococci suppression after chlorhexidine-thymol varnish treatments in schoolchildren. Acta Odontol Scand 1999;57:(3): 144-48

70. Joharji RM, Adenubi JO. Prevention of pit and fissure caries using an antimicrobial varnish: 9 mouth clinical evaluation. J. Dent 200129 (4): 247-54.

71. Baca P, Muñoz MJ, Bravo M, Junco P, Baca AP. Effectiveness of chlorhexidine-thymol varnish for caries reduction in permanent first molars of 6-7year-old children: 24-mouth clinical trial. Community Dent Oral Epidemiol 2002; 30(5):363-8.

72. Araujo AM, Naspitz GM, Chelotti A, Cai S. Effect of Cervitec on mutans streptococci in plaque and on caries formation on occlusal fissures of erupting permanent molars. Caries Res 2002 SepOct;36(5):373-6

73. Huizinga ED, Ruben JL, Arend J. Chlorhexidine and thymol release from a varnish system. J. Biol Buccale 1991 19(4):343-8

74. Petersson LG, Maki Y, Twetman S, Edwarsson S. Mutans streptococci in saliva and interdental spaces after topical applications of an antibacterial varnish in schoolchildren. Oral Microbiol Immunol 1991 Oct;6(5):284-7.

75. Twetman S Petersson LG. Effect of different chlorhexidine varnish regimens on mutans streptococci levels in interdental plaque and saliva. Caries Res 1997;31(3):189-93

76. Twetman S, Petersson LG. Comparison of afficacy of three different chlorhexidine preparations in decreasing the levels of mutans streptococci in saliva and interdental plaque. Caries Res 1998; 32(2):113-8

77. Pham Huy D, Rouveix B. Antisépticos y sesinfectantes. En: Farmacología Odontológica.1994. Masson. 16: 154-60

78. World Health Organization. Oral Health Surveys. Basic Methods 4th ed., Geneva, WHO 1997.

79. SPSS. SPSS 11.5 Syntax Reference Guide. SPSS Inc. 2002.

80. Ferrán Aranaz, M. SPSS para Windows. Programación y Análisis Estadístico. 1996 Mc- 
Graw Hill.

81. Baca P. Trabajo Original de investigación. Resumen: Influencia de la discontinuidad y del riesgo de caries en la eficacia de un programa de barniz de clorhexidina. Granada, 2003. 1-58

82. Powell LV. Caries prediction: a review of the literature. Community Dent Oral Epidemiol. 1998 Dec;26(6):361-71.

83. Gray MM, Marchment MD, Anderson RJ The relationship between caries experience in the deciduous molars at 5 years and in first permanent molars of the same child at 7 years. Community Dent Health. 1991 Mar;8(1):3-7

84. Li Y, Wang W. Predicting caries in permanent teeth from caries in primary teeth: an eight-year cohort study. J Dent Res 2002 Aug;81(8):561-6

85. Unkel JH, Fenton SJ, Hobbs G Jr, Frere CL. Toothbrushing ability is related to age in children. ASDC J Dent Child. 1995 Sep-Oct;62(5):346-8.

86. Von der Fehr FR, Löe $\mathrm{H}$ and Theilade Else. Experimental Caries in Man. Caries Res. 1970;4: 131-48.

87. Bossert WA. The Relation Between the Shape of the Occlusal Surfaces of Molars and the Prevalence of Decay. J Dent. Res. 1937;16: 63-7.

88. Reid DBW and Grainger, RM. Variations in the Caries Susceptibility of Children's teeth. Hum. Biol. 1955;27: 1-11.

89. Gibbons RJ and VanHoute J. Dental Caries. Ann Rev. Med. 1975;26: 121-36.

90. Ripa W, Leske S. and Varma O. Longitudinal Study of the Caries Susceptibility of Oclusal and Proximal Surfaces of First Permanent Molars. J. Public Heah Dentistry. 1988;48(1): 8-13.

91. Splieth C, Steffen H, Rosin M, Welk A. Caries prevention with chlorhexidine-thymol varnish in high risk schoolchildren Community Dent Oral Epidemiol. 2000 Dec;28(6):419-23
92. Carlos JP, Gittelsohn AM. Longitudinal Studies of the Natural History of Caries. II. Archs Oral Biol. 1965; 10: 739-51

93. Nikiforuk Gordon. Epidemiología de la Caries Dental. En: Caries Dental. Editorial Mundi Argentina C 2: 25-59 1986

94. Nikiforuk Gordon. La naturaleza de la sustancia dentaria. En: Caries Dental. Editorial Mundi Argentina C 4: 83-118. 1986

95. Legler Donald W. y Menaker Lewis. Definición, etiología, epidemiología e implicaciones clínicas de la caries dental. En: Menaker Lewis Bases Biológicas de la Caries Dental.. Salvat Barcelona 1986. Versión española.8: 223-60

96. Thylstrup A, Fejerskov O. Patología de la caries dental. En: Caries. Doyma. Edición en inglés 1986, en español 1988. 11: 170-96

97. Weyne S. Cariología. En: Operatoria Dental Procedimientos Preventivos y Restauradores. Baratieri Luiz N. Et al. Quintessence. Versión española de la segunda edición. 1993. 1: 1-42

98. Krasse B. Approaches to prevention. In: O’Brien W Y. et al. Proc. microbiol. Aspects of dental caries. Sp. Suppl. Microbiol. Abstracts. 1976;3: 867-76.

99. Zickert I, Emilson CG, Krasse B. Effect of caries preventive measures in children highly infected with the bacterium Streptococcus mutans. Arch Oral Biol 1982;27(10):861-68.

100. Zickert I, Emilson CG, Krasse B. Microbial conditions and caries increment 2 years after discontinuation of controlled antimicrobial measures in Swedish teenagers. Community Dent Oral Epidemiol 1987 Oct;15(5):241-4

101. Fennis-le YL, Verdonschot EH, Burgersdijk RC, Konig KG, van 't Hof MA. Effect of 6-monthly applications of chlorhexidine varnish on incidence of occlusal caries in permanent molars: a 3-year study. J Dent 1998 Mar;26(3):233-8. 
102. Forgie $\mathrm{AH}$, Paterson $M$, Pine $C M$, Pitts NB, Nugent $\mathrm{ZJ}$. A randomised controlled trial of the caries-preventive efficacy of a chlorhexidinecontaining varnish in high-caries-risk adolescents. Caries Res. 2000 Sep-Oct;34(5):432-9.

103. Almerich-Silla, JM, San Antonio- Morat, I, Tatay-Vivó, V y Ortolá-Siscar, JC. Eficacia de un barniz de Clorhexidina y Timol, al $1 \%$, en la reducción de los niveles de Mutans Streptococci y Lactobacilli en saliva. Un ensayo clínico. RCOE, 2000;1 5(6):643-9.

104. Haukali G, Poulsen S. Effect of a varnish containing chlorhexidine and thymol (Cervitec) on approximal caries in 13- to 16-year-old schoolchildren in a low caries area. Caries Res. 2003 May-Jun; 37(3): 185-9

105. Luoma H. Chloehexidine solutions, gel and varnishes in caries prevention. Proc Finn Dent Soc. 1992; 88(3-4):147-53.

106. Rioboo R. Estudio de la microbiota bucal. En: Rioboo R. Odontología Preventiva y Odontología Comunitaria. Ediciones Avances. Madrid. 2002:(3)77-14
107. Mouton Christian, Robert Jean-Claude. Placa dental. En: Bacteriología bucodental. Masson Barcelona 1995. 2: 19-33.

108. Arends J, Ruben J. Chlorhexidine CHX release by dentin after varnish treatment. Caries Res 1993:206-40. Abstracts 88

109. Netuschil L, Weiger $R$, Preisler $R$, Brecx $M$. Plaque bacteria counts and vitality during chlorhexidine, meridol and listerine mouthrinses. Eur J Oral Sci 1995 Dec;103(6):355-61

110. le YL, Schaeken MJ. Effect of single and repeated application of chlorhexidine varnish on mutans streptococci in plaque from fissures of premolar and molar teeth. Caries Res. 1993;27(4):303-6

\section{CORRESPONDENCIA}

García-Santos María C.

Facultad de Odontología

Departamento Estomatología IV

Universidad Complutense de Madrid

C/ Ramón y Cajal, s/n.

28040 Madrid 
